\title{
Mapping Retinotopic Structure in Mouse Visual Cortex with Optical Imaging
}

\author{
Sven Schuett, Tobias Bonhoeffer, and Mark Hübener \\ Max-Planck-Institut für Neurobiologie, D-82152 Martinsried, Germany
}

\begin{abstract}
We have used optical imaging of intrinsic signals to visualize the retinotopic organization of mouse visual cortex. The functionally determined position, size, and shape of area 17 corresponded precisely to the location of this area as seen in stained cortical sections. The retinotopic map, which was confirmed with electrophysiological recordings, exhibited very low interanimal variability, thus allowing averaging of maps across animals. Patches of activity in area 17 were often encircled by regions in which the intrinsic signal dropped below baseline, suggesting the presence of strong surround inhibition. Singleunit recordings revealed that this decrease of the intrinsic signal
\end{abstract}

indeed correlated with a drop of neuronal firing rate below baseline. The averaged maps also greatly facilitated the identification of extrastriate visual activity, pointing to at least four extrastriate visual areas in the mouse. We conclude that optical imaging is ideally suited to visualize retinotopic maps in mice, thus making this a powerful technique for the analysis of map structure in transgenic animals.

Key words: visual cortex; optical imaging; brain mapping; retinotopy; map formation; cortical magnification factor; lateral inhibition; mice
The visual cortex of the mouse consists of a number of anatomically (Olavarria et al., 1982; Olavarria and Montero, 1989) and functionally (Dräger, 1975; Wagor et al., 1980) distinct areas. Mouse primary visual cortex seems to lack any obvious parcellation into functional domains, such as ocular dominance or orientation columns, which are a prominent feature in the visual cortex of many higher mammals (Hubel and Wiesel, 1977; Blasdel and Salama, 1986; Ts'o et al., 1990; Hübener et al., 1997), but it has been shown that it does contain an ordered retinotopic map (Dräger, 1975; Wagor et al., 1980). However, the detailed layout of this map and the distribution of the cortical magnification factor (CMF), as well as the inter-animal variability, have not been studied systematically.

There is also disagreement on the number and organization of extrastriate areas in rodent visual cortex in general. Although a number of electrophysiological and anatomical mapping studies suggest that rodent extrastriate cortex consists of many (up to 10) distinct visual areas (Montero, 1973; Montero et al., 1973a,b; Espinoza and Thomas, 1983; Olavarria and Montero, 1984; Thomas and Espinoza, 1987; Coogan and Burkhalter, 1990, 1993; Montero, 1993), others have put forward the idea that the primary visual cortex is surrounded by just one or two areas, which are composed of multiple processing modules comparable to those found in primate visual cortex (Kaas et al., 1989; Malach, 1989; Rumberger et al., 2001).

Because the high resolution mapping of cortical areas with electrode recordings is very tedious and error prone, we used

\footnotetext{
Received Dec. 26, 2001; revised April 29, 2002; accepted May 3, 2002.

This work was supported by the Max-Planck Gesellschaft and by the Graduate Program GRK 267 of the Deutsche Forschungsgemeinschaft. We thank Iris Kehrer for technical assistance as well as Valentin Nägerl and Miguel Vaz Afonso for comments on this manuscript.

Correspondence should be addressed to Dr. Mark Hübener, Max-Planck-Institut für Neurobiologie, Am Klopferspitz 18A, D-82152 Martinsried, Germany. E-mail: mark@neuro.mpg.de.

Copyright (C) 2002 Society for Neuroscience $\quad 0270-6474 / 02 / 226549-11 \$ 15.00 / 0$
}

optical imaging of intrinsic signals (Grinvald et al., 1986) to address these questions.

A further motivation for establishing the method of optical imaging in mouse visual cortex is the increased use of transgenic mice. Mapping the retinotopic structure of mouse visual cortex is particularly interesting because a central question of developmental neurobiology is the establishment of topographically ordered projections in the brain (Sperry, 1963; Tessier-Lavigne and Goodman, 1996; Flanagan and Vanderhaeghen, 1998). The ease of use and the small inter-animal variability of mapping mouse visual cortex with optical imaging allows for the efficient screening of genetic factors that contribute to the establishment of ordered projections and areal specification in the cerebral cortex.

\section{MATERIALS AND METHODS}

The experiments were performed in 14 7- to 14 -week-old C57BL/6 as well as mixed background C57BL/6 $\times \mathrm{SV} / 129 \mathrm{~J}$ mice. All procedures were performed in accordance with local government rules and the guidelines of The Society for Neuroscience.

Surgery. Mice were anesthetized with a combination of urethane und ketamine (initial anesthesia, 130-140 mg/kg ketamine, i.m., $1500 \mathrm{mg} / \mathrm{kg}$ urethane, i.p., maintained by continuous intraperitoneal infusion: 120 $\mathrm{mg} \cdot \mathrm{kg}^{-1} \cdot \mathrm{hr}^{-1}$ ketamine, $130 \mathrm{mg} \cdot \mathrm{kg}^{-1} \cdot \mathrm{hr}^{-1}$ urethane). This combination of anesthetics was found to be crucial for reproducible highquality imaging and long-term stability of physiological conditions. In particular, to reliably image retinotopic maps, the pupils should not be dilated by the anesthetics or other drugs. We were not able to image these maps when using halothane anesthesia alone or when atropine was applied systemically in pilot experiments on rats, presumably because the pupils were dilated. A mixture of $35 \%$ glucose and $65 \%$ saline was infused intraperitoneally at $3 \mathrm{ml} \cdot \mathrm{kg}^{-1} \cdot \mathrm{hr}^{-1}$ to prevent dehydration. The animal was initially positioned stereotaxically using mouth and ear bars, with the mouth bar positioned $3 \mathrm{~mm}$ below the interaural line. The skin above the skull was cut open, and a few drops of silicone oil were immediately applied to the bone, thereby maintaining it sufficiently transparent to allow for optical imaging through the intact skull. The skull was then attached to a head holder with a mixture of glass beads and tissue glue (Histoacryl, Braun), and the mouth and ear bars were removed. For electrical recording experiments, a trepanation of $4 \times 4$ $\mathrm{mm}$ above the visual cortex was performed after optical imaging. During surgery, the eyes were covered with eye protection cream (Isopto-Max, 
Alcon Thilo), which was replaced by silicone oil to prevent drying of the cornea during optical imaging.

In most experiments, the animals breathed spontaneously with a flow of pure oxygen $(0.51 / \mathrm{min})$ directed to the nose. For experiments with both optical and electrical recordings, a small tubing was attached to the nose, and the animals were respirated artificially (100-150 cycles per minute). In these cases, anesthesia was maintained additionally by $50 \%$ $\mathrm{N}_{2} \mathrm{O}$ and $50 \% \mathrm{O}_{2}$. We monitored the heart rate continuously and adjusted the depth of anesthesia to maintain a rate of 350-500 beats per minute. With the above procedures, we were able to record optical signals for $\sim 20 \mathrm{hr}$ and single-unit responses for up to $24 \mathrm{hr}$.

In three mice, which were not used for optical imaging, we dilated the pupils with atropine to determine the optic disk projection, which was found to vary only little between animals (elevation: $+32.7 \pm 1.5^{\circ} \mathrm{SEM}$; azimuth: $55.0 \pm 2.0^{\circ} \mathrm{SEM} ; n=6$ eyes from three mice).

Imaging and visual stimulation. For visual stimulation we backprojected the stimuli with a video beamer onto a curved plastic screen covering the visual field in horizontal dimension from -50 to $+150^{\circ}$ measured relative to the vertical midline and between about -55 to $+50^{\circ}$ relative to the optic disk projection in vertical dimension. We stimulated at several positions in the visual field using moving square wave gratings, which changed their orientations randomly every $0.6 \mathrm{sec}$ (spatial frequency: 0.05 cycles per degree; speed: 2 cycles per second). These square-shaped stimuli were presented in a random manner at adjacent positions (for optical imaging: seven columns and three rows of stimuli, $25^{\circ}$ side length; for combined optical and electrical recording: five columns, three rows, $35^{\circ}$ side length). Each $6 \mathrm{sec}$ stimulus presentation at one position was followed by a blank screen for $8 \mathrm{sec}$. To maximize the on-response, we stimulated with a minimal luminance of $1.25 \mathrm{~cd} / \mathrm{m}^{2}$ (maximal luminance: $205 \mathrm{~cd} / \mathrm{m}^{2}$ ) for blank screen and background intensity. In five experiments, computer-controlled shutters allowed independent stimulation of the ipsilateral and contralateral eyes. In all other experiments, we stimulated only the contralateral eye.

For optical imaging, the cortex was illuminated with monochromatic light of $707 \mathrm{~nm}$ wavelength. Images were captured using a cooled slowscan CCD camera (ORA 2001, Optical Imaging, Germantown, NY), focused $700 \mu \mathrm{m}$ below the cortical surface. Four "first frames" (Bonhoeffer and Grinvald, 1996) and 10 frames of $600 \mathrm{msec}$ duration were collected immediately before and during each $6 \mathrm{sec}$ stimulus presentation, respectively.

Electrophysiology. In three animals, we recorded single units extracellularly after the optical imaging. The electrode recording sites were placed within area 17 , which was determined previously by optical imaging. Using a similar stimulus as described above (five columns, three rows, $35^{\circ}$ side length), we recorded the spike response of 22 single cells discriminated by their waveforms (Brainware, Oxford, UK). Instead of randomizing orientation, we optimized for orientation, spatial frequency, and direction to elicit a maximal firing rate.

Analysis. All images were "first frame-corrected"; i.e., from each image stack obtained during presentation, we subtracted the four first frames taken immediately before stimulus presentation. The resulting images were blank-corrected by subtracting the images acquired without stimulus from all images. In addition, the images were processed by a blood vessel extractor to remove blood vessel-related artifacts. This method significantly improved the intertrial correlation (Schuett et al., 2000). Twelve-bit digitized camera data were range-fitted such that for the single-condition maps the 1 or $3 \%$ most responsive pixels (least responsive pixels) of the entire set of images were set to black (white) for all DC-corrected or high-pass-filtered images, respectively. To avoid erroneous DC correction caused by large activated areas, which will slightly increase the mean of the intrinsic signal, we corrected for DC shift in each image using the mean of only those pixels, which varied $<1$ SD of the blank response from its mean.

For quantification and alignment, all images where low-pass filtered with a Gaussian kernel of $99 \mu \mathrm{m}$ half-width. Because quantification requires a high signal-to-noise ratio, we used 7(5) of 11(9) animals with the best signal-to-noise ratio for the quantification with stimulation via the contralateral (ipsilateral) eye.

The region of the primary visual cortex was determined by thresholding the maximum intensity projection of the intrinsic signal, i.e., the light absorption, $5 \mathrm{SD}$ of the blank response above the mean of the blank. In some cases, extrastriate areas lateral of area 17 had to be excluded by hand. The position of these lateral areas could be easily delineated by both shape and intensity. All subsequent computations were fully automated.
Both for averaging of single-condition maps across animals and for quantification, we aligned all sets of single-condition maps imaged in different animals using the eight most prominent patches (see Fig. $1 A$, stimuli 2-5 in $b$ and $c$ ) elicited by visual stimulation as reference points to determine translation and rotation parameters. Patch position was defined by the center of mass of the intrinsic signal in the primary visual cortex after thresholding the map $5 \mathrm{SD}$ above its mean. The CMF was determined by the mean distance to neighboring patches elicited by adjacent visual stimuli.

To visualize the overall retinotopic organization across the cortical surface, we color-coded visual field position: in the "peak position projection," each pixel was assigned the color corresponding to the stimulus (see Fig. $1 A$ ) eliciting the strongest response at this pixel. Because of this "winner takes all" algorithm, the peak position projection contains distinct borders between cortical regions responding preferentially to different stimuli. This type of coding can lead to an erroneous representation of cortical retinotopy, when patches activated by adjacent stimuli overlap extensively and when the intensity of the intrinsic signal differs between patches. To avoid this, we additionally used the "average position projection": in this coding scheme, we determined the average corresponding stimulus position at each pixel by computing the weighted mean across all stimuli eliciting an excitatory response (defined as intrinsic signal strength above the mean of the blank response). The color corresponding to the averaged stimulus position is then assigned to this pixel.

To mask regions without a cortical response, color saturation was chosen to code for the maximum intensity projection of the intrinsic signal across all single-condition maps for both the peak and the average position projections.

Histology. The size and shape of area 17 was determined in two animals after optical imaging, using both SMI-32 antibodies (Sternberger Monoclonals, Lutherville, MD) and cytochrome oxidase staining as anatomical markers for area 17 (Duffy et al., 1998). To this end, the animal was euthanized and perfused with $0.1 \mathrm{M}$ PBS followed by $2 \%$ paraformaldehyde in $0.1 \mathrm{M}$ PBS. The brain was removed and postfixed for $1 \mathrm{hr}$. The cortex was dissected and flattened, and $50 \mu \mathrm{m}$ tangential sections were cut on a freezing microtome. For alignment with the functional maps, the section containing the pial surface was cut at a thickness of $250 \mu \mathrm{m}$ to enable the visualization of the superficial blood vessel pattern. Every other regular section, as well as the thick section, was stained for cytochrome oxidase according to procedures described by Wong-Riley (1979). The remaining sections were stained with the SMI-32 antibody according to Duffy et al. (1998).

To compare the staining pattern with the imaged maps, we first aligned the superficial blood vessel pattern visible in the thick section with the blood vessel image taken before optical imaging (Bosking et al., 1997). Prominent blood vessels could be readily identified in both images, which were also used to correct for tissue shrinkage caused by fixation. The thin sections were then aligned with the thick section using the pattern of vertical blood vessels that was present in all sections. For display, the images of the stained sections were intensity clipped.

\section{RESULTS}

We imaged the intrinsic signal in mouse visual cortex evoked by stimulation at different positions in the visual field. To elicit a maximal cortical response, we presented a moving grating of changing orientations within a window of $25 \times 25^{\circ}$ at each position. By using a curved screen, we were able to stimulate a large part of the visual field of the mouse (Fig. 1A).

Each single-condition map corresponding to one retinotopic stimulus exhibits a prominent patch of activity that is adjacent (and at the same time overlapping) to the primary patch corresponding to an adjacent stimulus in the visual field (Fig. 1C), indicating that an ordered retinotopic map should be reconstructable from images recorded with this stimulation paradigm. The activity maps proved to be highly reproducible over the course of an experiment (Fig. 1D). The intertrial correlation coefficient (0.71; three randomly picked animals) is comparable to the correlation coefficient for orientation preference maps in cat visual cortex (0.73; three animals), which can be regarded as a benchmark for optical imaging. This high intertrial correlation also 
suggests that eye movements do not impede imaging of retinotopic maps.

\section{Retinotopic organization of area 17}

Using stereotaxic coordinates as well as anatomical markers for area 17, we confirmed that the principal patches are located within the primary visual cortex. We determined the extent of area 17 with a maximum intensity projection of the intrinsic signal across all imaged single-condition maps and aligned the resulting map with both cytochrome oxidase-stained and SMI32-stained (Duffy et al., 1998) sections using the superficial blood vessels as landmarks (Fig. 1E, top panels). Both the size and shape of the imaged and the stained areas are in very good agreement (Fig. 1E, bottom panels), indicating that the primary patches are located in area 17.

To illustrate the overall organization of the entire retinotopic map, we used a color code for stimulus position (Fig. $1 A, F$ ). Each pixel within the map was assigned the color of the stimulus position, which had elicited the largest response at that point in the cortex (peak position projection; see Materials and Methods). To mask cortical regions devoid of activation, we used the maximum intensity projection of the intrinsic signal across all singlecondition maps for color saturation. Hence, nonresponsive regions remain dark. In this manner, we obtained a multicolored region at the center of the image, exhibiting a continuous retinotopic progression, in both vertical and horizontal directions. As demonstrated by the correspondence between the maximum intensity projection of the intrinsic signal with the anatomical staining of area 17, this colored patch reveals the retinotopic organization of the primary visual cortex. Although we also detected activity in extrastriate areas, the intrinsic signal strength is much smaller in these regions. Therefore, extrastriate areas are not visible in this color-coded retinotopic map.

Because the retinotopic maps from different animals were very similar, we reasoned that it should be possible to average the single-condition maps across animals. To this end, we first aligned maps from different animals using the center of mass of the eight strongest patches as reference points for translation and rotation. In addition, we standardized the experiments to control for variations in intrinsic signal strength, i.e., the mean across each image was set to 0 and the spatial SD was set to 1 .

The resulting maps were then averaged across seven animals with the highest signal-to-noise ratio. Both the averaged singlecondition maps (Fig. 2A) and the averaged color-coded retinotopic maps (Fig. $2 B$ ) are similar to the data obtained from the individual animal presented in Figure 1. The possibility of reconstructing mouse retinotopy from the averaged single-condition maps across animals indicates that both the variance caused by the method and the functional differences between animals are small.

Averaging of single-condition maps across animals also enabled us to directly visualize inter-animal variability by computing maps that display the spatial distribution of the SEM (Fig. 2C). Overall, these maps are very dark (despite the fact that, for better visibility, they were scaled by a factor of 5 in comparison with the single-condition maps in Fig. $2 A$ ), indicating that there is little variation between animals. In addition, we quantified the interanimal variability by computing the correlation coefficient between the single-condition maps from different animals. The high correlation coefficient between maps from different animals $(0.61 \pm 0.10$ SEM; seven animals, 21 maps per animal) substan- tiates that the inter-individual variation between retinotopic maps is low.

To confirm the retinotopic maps obtained with optical imaging in the primary visual cortex, we recorded 22 single units in three animals using the same visual stimuli for optical and electrical recordings. The locations of the tracks within the functional maps were determined with the help of the superficial blood vessel pattern. The positions of the center of the receptive fields were defined by the stimulus causing the strongest response. The histogram of the distance between receptive field positions determined optically and electrically validates the retinotopic maps (Fig. 2D; plots per unit, see Fig. 4A).

In summary, the imaged retinotopic order in the primary visual cortex and the high intertrial and inter-animal correlation, as well as the correspondence between electrical and optical recordings, show that our paradigm is well suited to image the retinotopic map in mouse visual cortex.

\section{Cortical magnification factor}

Having obtained the retinotopic map in area 17, we were able to measure the spatial distribution of the CMF, a measurement that is difficult to accomplish using conventional methods. For this purpose, we used single-condition maps from the seven animals with the highest signal-to-noise ratio.

The CMF is defined as the scaling factor that relates a distance in the visual field (in degrees of visual angle) to the cortical distance (in millimeters) of the corresponding cortical representation (Daniel and Whitteridge, 1961). In our case, the reference length in the visual field is given by the center distance between two adjacent visual stimuli, i.e., $25^{\circ}$. The corresponding cortical distance was defined as the difference between the centers of mass of the activated patches in primary visual cortex corresponding to each stimulus. For this purpose, the single-condition maps were thresholded $5 \mathrm{SD}$ above the mean of the blank response.

Figure $3 A$ displays the averaged retinotopic map in the primary visual cortex in retinal coordinates relative to the averaged optic disk projection. The crosses indicate the positions (and SEM) of the centers of mass of each patch for ipsilateral and contralateral eye stimulation (green and red crosses, respectively). This map confirms that the retinotopic organization across the primary visual cortex is maintained. Because the spacing of the visual stimuli is uniform, the CMF is directly proportional to the distance between positions, corresponding to adjacent visual stimuli.

Accordingly, we defined the horizontal and vertical CMF for a given stimulus position as the mean cortical distances corresponding to horizontal and vertical stimuli, respectively. To illustrate the distribution of the CMF across the visual field, we used a schematic of the stimulus pattern and assigned to each stimulus position the corresponding $\mathrm{CMF}$, coded by lightness (Fig. 3B,C). Figure $3 A$ shows that the gradient of the CMF is relatively shallow in contrast to higher mammals, such as primates, varying only by a factor of $\sim 2$. It is also obvious that the horizontal and the vertical CMF are different (Fig. $3 B, C$ ). Although both the vertical and the horizontal CMF peak in the central visual field, the exact peak positions as well as the shape of the gradients do not match. In addition, the vertical CMF is significantly larger than the horizontal CMF (vertical magnification: $24.8 \pm 5.9$ $\mu \mathrm{m} /{ }^{\circ}$; horizontal magnification: $14.5 \pm 5.4 \mu \mathrm{m} /{ }^{\circ} ; p=0.034 ; t$ test; $n=7)$.

The data presented above were obtained with monocular stim- 
A

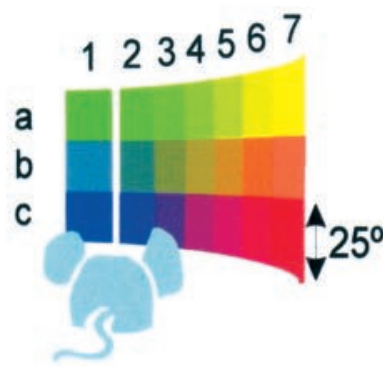

B

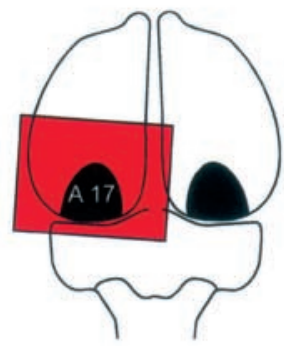

E

Anatomy

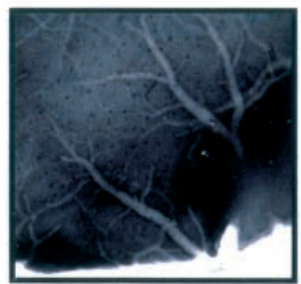

Blood Vessel Pattern

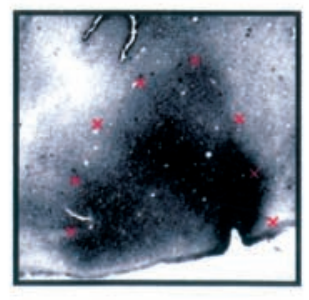

SMI-32 Staining
C 12 3 4 5 6 7

a

b
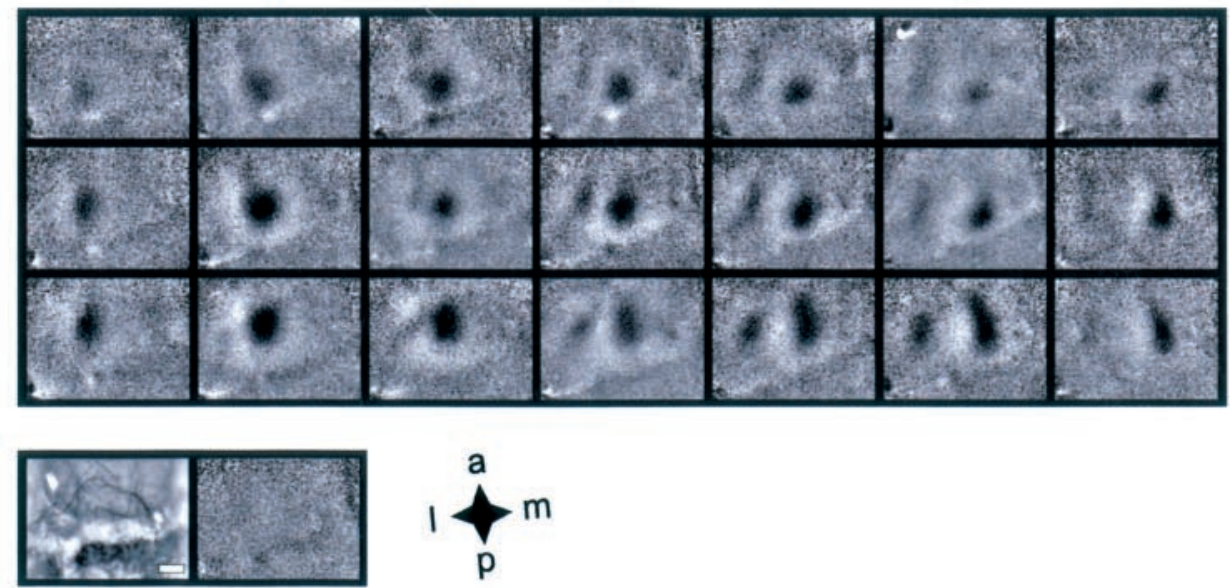

D

b

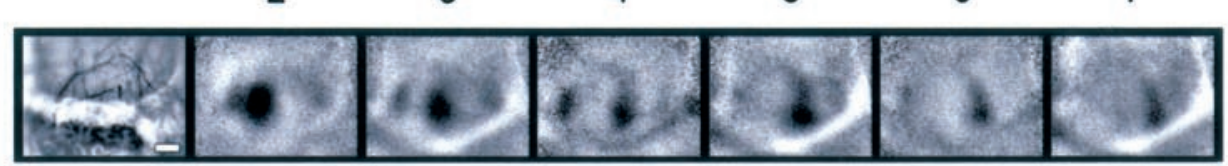

b

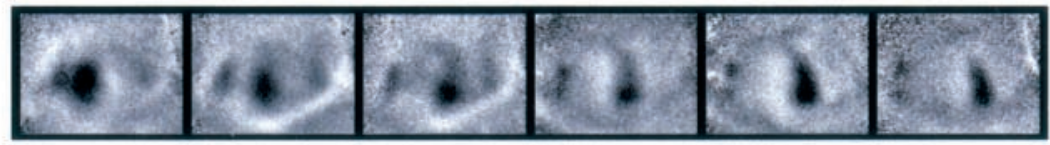

\section{F}

Imaging

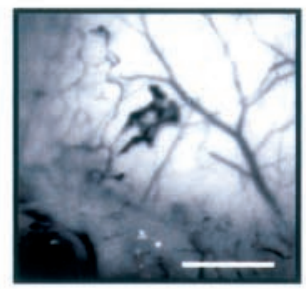

Blood Vessel Pattern

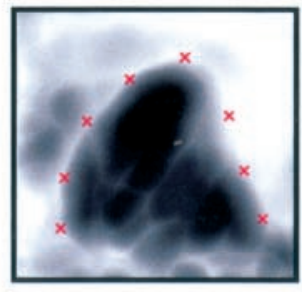

Activity Map
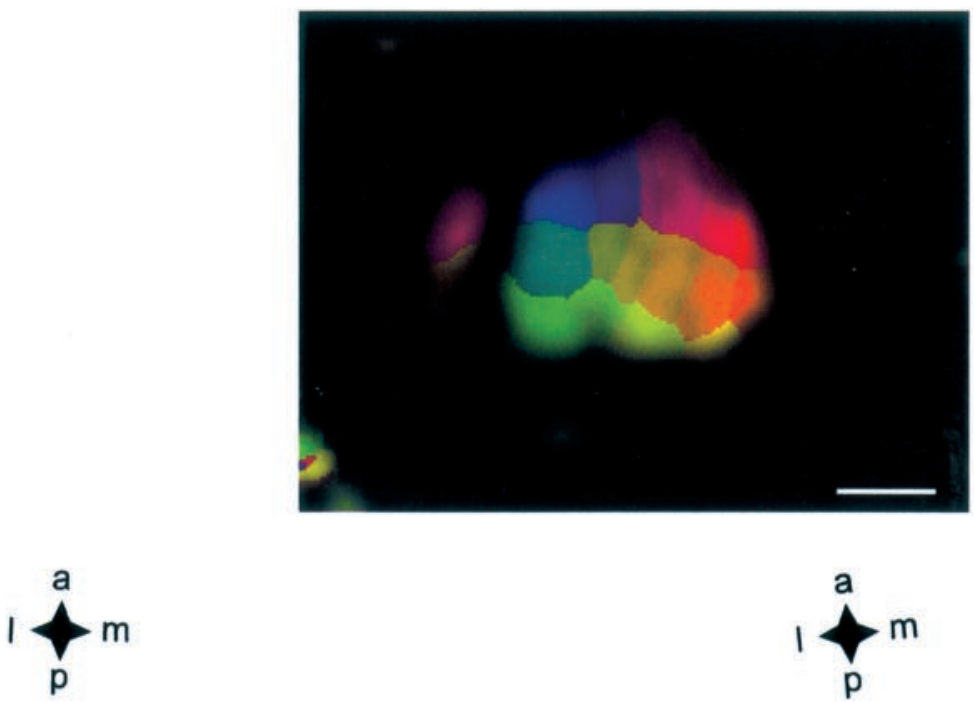

Figure 1. Imaging cortical retinotopy in mice. $A$, For retinotopic stimulation, we used square-shaped windows of gratings $\left(25^{\circ}\right.$ side lengths) at adjacent positions within the visual field. The color code representing stimulus position was used to generate the color-coded retinotopic maps. The white line indicates the vertical midline. $B$, Schematic of the imaged cortical region as indicated by the red window, which contains a rough outline of area $17 . C$, Blank corrected single-condition maps from one animal, with each map corresponding to a stimulus in $A$, according to the indices. All maps are scaled and clipped to the same absolute values. The cortical blood vessel pattern imaged through the translucent skull is shown at the bottom left. The map next (Figure legend continues.) 


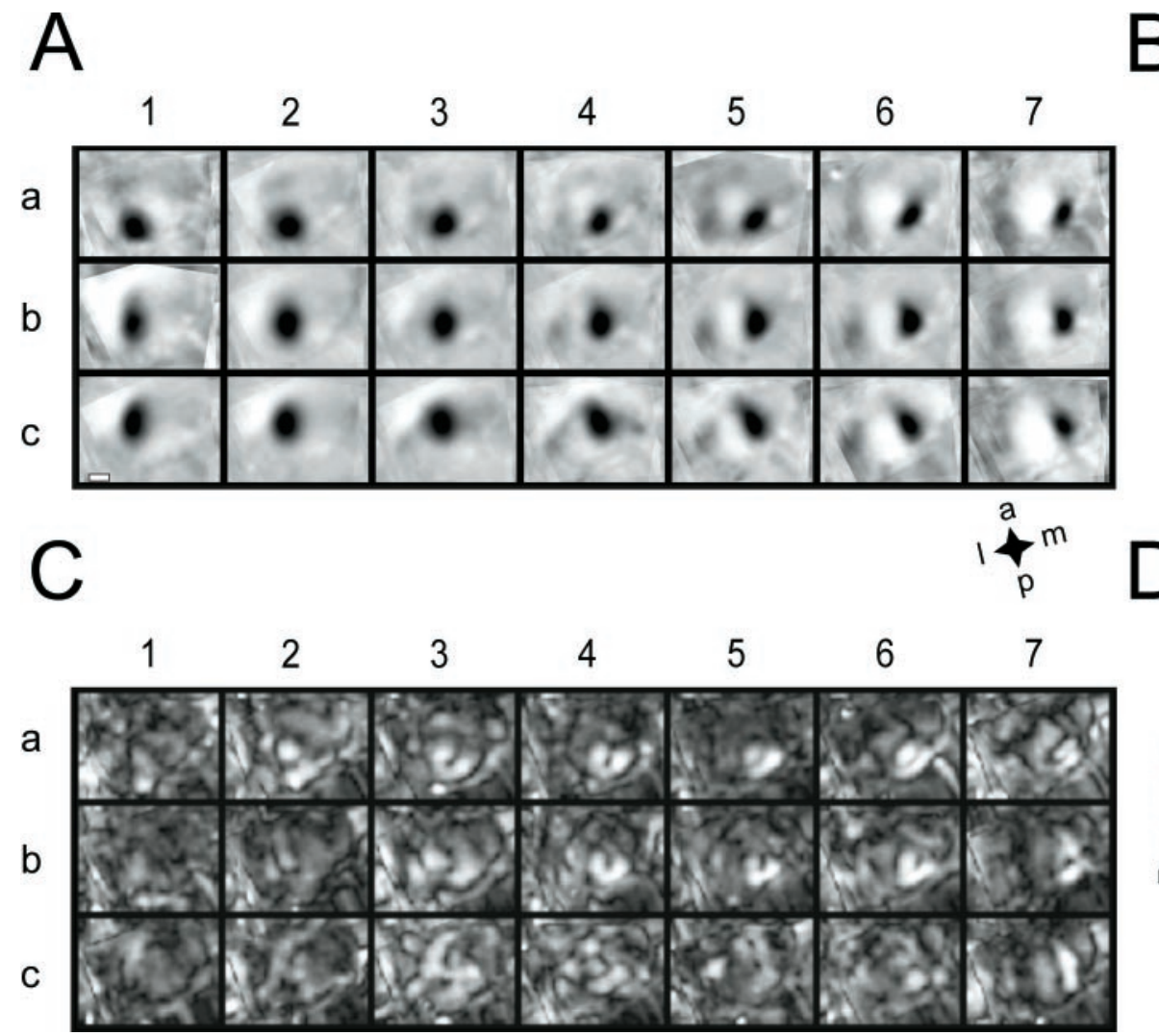

\section{B}
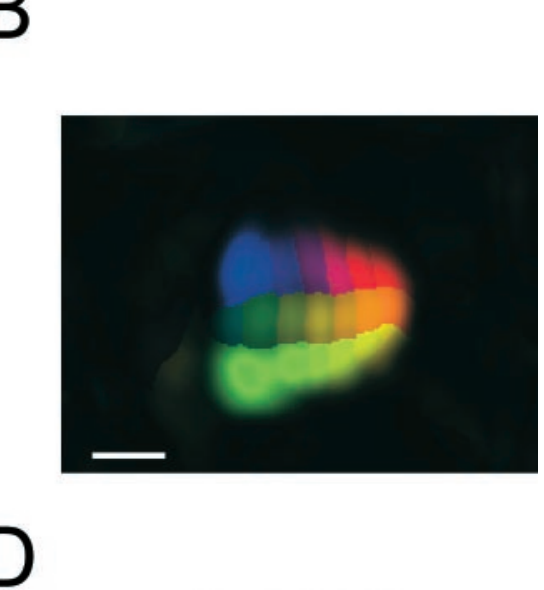

\section{Excitation}

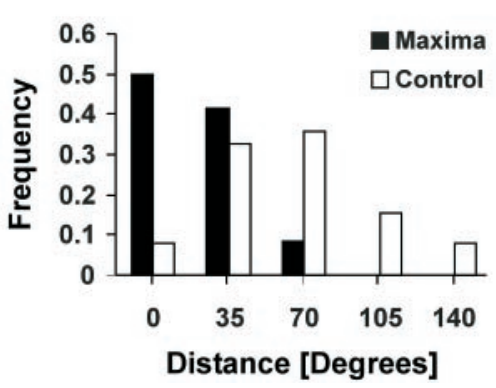

Figure 2. Averaging maps across animals. $A$, Single-condition maps averaged across seven animals. Indices denote stimulus position according to Figure $1 A$. $B$, Color-coded retinotopic map based on the averaged single-condition maps. $C$, Spatial distribution of the SEM for each map. For better visibility, the SEM maps are scaled by a factor of 5 in comparison with the single-condition maps in $A$. The overall dark appearance of the maps indicates that the variability between animals is low. $D$, Histogram of the distances between receptive field positions (i.e., the maximal response) determined electrically and optically based on recordings from 22 neurons (black bars). The control distribution (white bars) was calculated from randomly assigning receptive field positions for 22 pairs and calculating the histogram of their distances. Note that $50 \%$ of the electrically measured receptive field positions coincide with the optically determined position. Scale bars, $1 \mathrm{~mm}$.

ulation of the contralateral eye. In five animals, we additionally stimulated monocularly through the ipsilateral eye and compared the position of the cortical regions activated by either eye (Fig. $3 A$ ). As expected, only the two central columns of stimuli yielded a response after the ipsilateral eye was stimulated. We found no significant differences between the patch positions for each stimulus when comparing ipsilateral and contralateral eye stimulation for the two central columns of stimuli. ( $p=0.19 ; t$ test; $n=5)$. This indicates that the retinotopic order and exact position of the cortical projections driven by the ipsilateral eye correspond precisely to projections driven by the contralateral eye.

Alternating monocular stimulation of both eyes enabled us to compute ocular dominance maps in these animals. We failed to detect any clustering with respect to ocular dominance. Similarly, no clustering for orientation preference or selectivity, expanding stimuli, or on versus off responses was detectable. This observation is in line with electrophysiological studies, which have provided weak evidence, at most, for the clustering of response properties in mouse visual cortex (Dräger, 1975; Mangini and Pearlman, 1980; Metin et al., 1988). A recent comprehensive electrophysiological study in rat visual cortex also failed to detect any clustering of orientation preferences (Girman et al., 1999).

\section{Lateral inhibition}

The single-condition images (Fig. $1 C$ ) and the averaged maps (Fig. $2 A$ ) exhibit not only a principal dark patch but also a light rim partly surrounding the dark patch. This increase in light reflectance corresponds to a reduction of the intrinsic signal

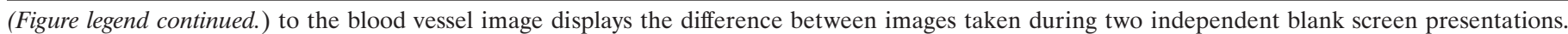

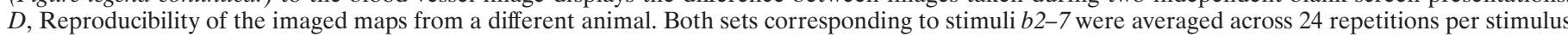

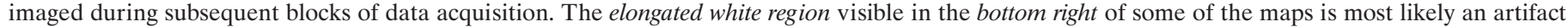

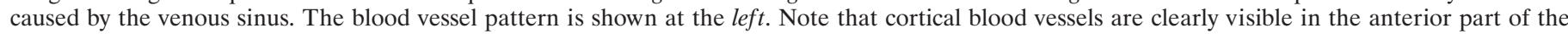

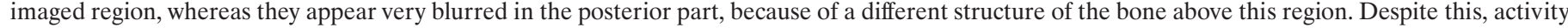

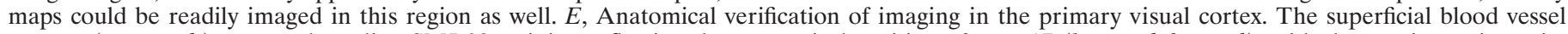

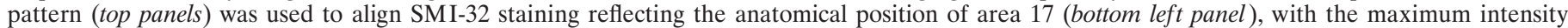

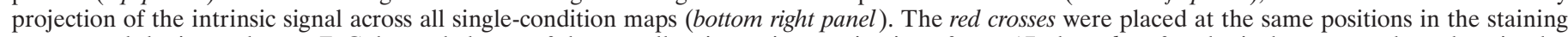

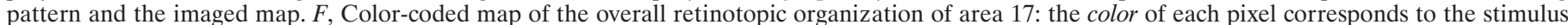

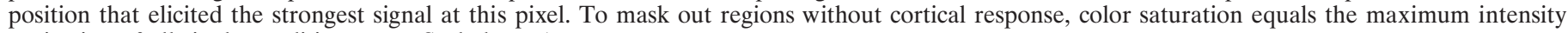
projection of all single-condition maps. Scale bars, $1 \mathrm{~mm}$. 
A

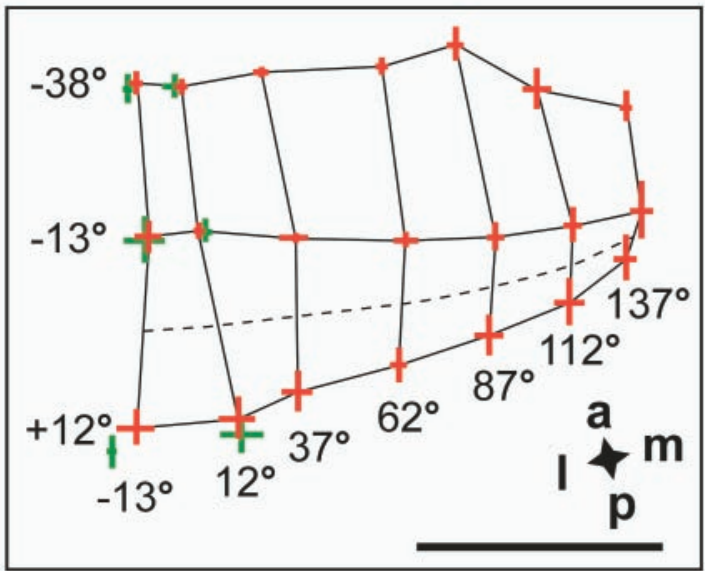

B

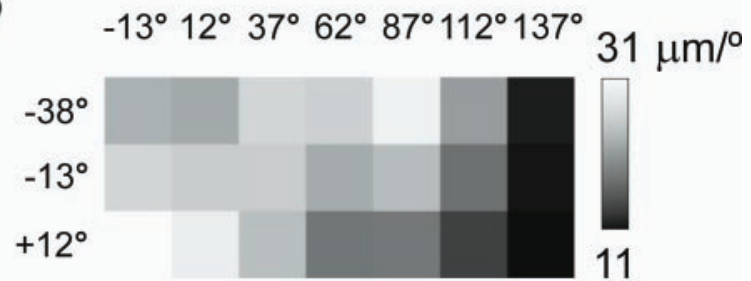

C



Figure 3. Distribution of the CMF in area 17. A, Layout of the averaged retinotopic map in area 17. Values to the left denote elevation relative to the optic disk projection, and values at the bottom indicate azimuth relative to the vertical midline (which coincides with the midline between the two optic disk projections). The gridlines display isoelevation and isoazimuth lines for the contralateral eye. The approximate position of the horizontal meridian (Wagor et al., 1980; Dräger and Olsen, 1981) is indicated by the dashed line. The red (contralateral eye) and green (ipsilateral eye) crosses denote the averaged positions $(n=7$ mice) of the centers of mass of the primary patches in area 17 . The lengths of the crossing bars indicate 1 SEM in vertical and horizontal direction. Scale bar, $1 \mathrm{~mm} . B, C$, Distribution of the CMF along the vertical $(B)$ and horizontal $(C)$ axes for each stimulus position (conventions as in $A$ ) coded by lightness.

below baseline values obtained in response to a blank stimulus. The detailed shape of this lighter region is somewhat variable, but in general it is particularly pronounced for peripheral stimuli. It is not confined to area 17 but might extend into extrastriate areas, particularly for central stimuli. This lighter surround region is not a filtering artifact, because the images have been corrected only for a DC shift without any additional high-pass filtering. We hypothesized that this light surround region could be an optical correlate of lateral inhibition, which has been reported in singlecell studies in mouse visual cortex (Mangini and Pearlman, 1980; Simmons and Pearlman, 1983).

To test this assumption, we recorded single units after optical imaging. A neuron was assigned an inhibitory surround if during presentation of a grating at any position its firing rate dropped below spontaneous levels recorded during presentation of the blank. According to this criterion, the receptive fields of 17 of 22 neurons recorded in the primary visual cortex exhibited an inhibitory surround. To compare the electrical and optical recordings, we measured the magnitude of the intrinsic signal within each single-condition map at the cortical position of the electrode tracks. Similarly, a drop of the intrinsic signal strength below the blank response (i.e., a relative increase in light reflectance) was taken as evidence for inhibition. We plotted the electrical and optical response by coding the firing rate or the amplitude of the intrinsic signal as the radius of circles corresponding to the different stimulus positions (Fig. 4A). For this analysis, only single units exhibiting lateral inhibition were chosen. The electrical and optical recordings exhibit similar positions for the "receptive field centers" and the lateral inhibitory regions (Fig. 4A). We quantified this coincidence by comparing the distributions of the distances between the electrically and optically determined positions with a randomized control. Most (83\%) of the distances between electrically and optically determined receptive field centers are at most $35^{\circ}$ apart from each other in visual space. For inhibition, $59 \%$ of the positions of the strongest inhibition are maximally $35^{\circ}$ in visual space apart from each other (Fig. $4 B$ ). It is to be expected that the coincidence of the inhibitory peaks is lower than the correlation of the centers of the receptive fields, because the inhibitory surround is more broadly spread out than the excitatory patches and because it has a lower signal-to-noise ratio. Despite this, the distribution of the distances for inhibition is clearly shifted toward smaller values when compared with the control distribution, which was generated on the basis of randomized pairs of positions.

The observed correlation between the decrease in the intrinsic signal and the electrically recorded lateral inhibition provides strong evidence that the intrinsic optical signal is a monotonous function of the firing rate. Therefore, a drop of the intrinsic signal below baseline, which is defined as the response to the blank screen, indicates neuronal inhibition.

\section{Extrastriate cortical areas}

We found that in addition to the strong response in the primary visual cortex, smaller patches are visible in extrastriate cortical areas in the single-condition images (compare Fig. $1 C$ ). Because the strong response within the primary visual cortex tends to overshadow these additional patches, we used a strong high-pass filter to reveal peaks of lower intensity and smaller size in the averaged single-condition maps (Fig. $5 A$ ).

Lateral, anterior, and medial to area 17 , small patches are visible in several of the averaged single-condition maps, as indicated in Figure $5 A$ by colored arrows. Note that patches in extrastriate areas are visible in only some of the single-condition maps. This could indicate that only a portion of the visual field is represented in these areas or that for some stimuli in some areas the activated cortical regions is too small to be reliably detected by optical imaging. In addition, because of the limited spatial resolution of optical imaging, some of the patches in extrastriate cortex might in fact correspond to activity in multiple small extrastriate areas. Again, we color-coded retinotopic position using the high-pass-filtered single-condition maps (Fig. $5 B$ ). To avoid overshadowing of extrastriate areas by the strong response 


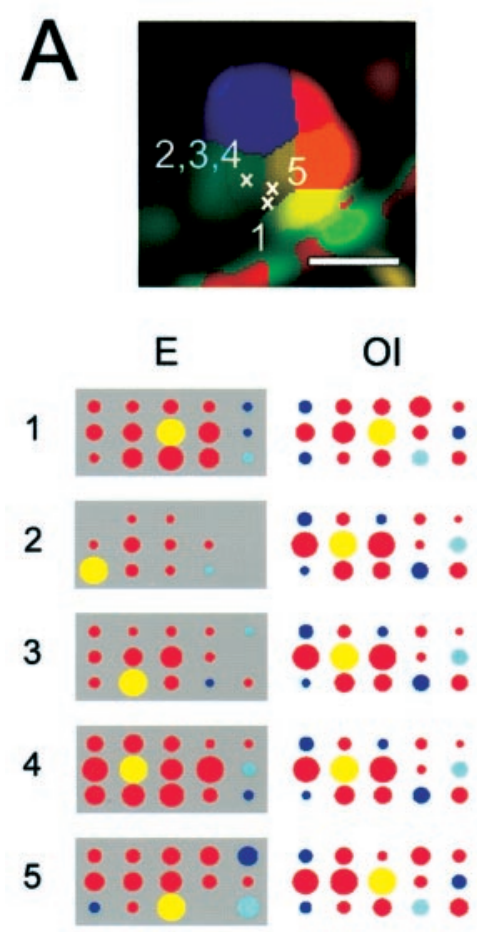

Peak Excitation

- Excitation

- Inhibition

Peak Inhibition
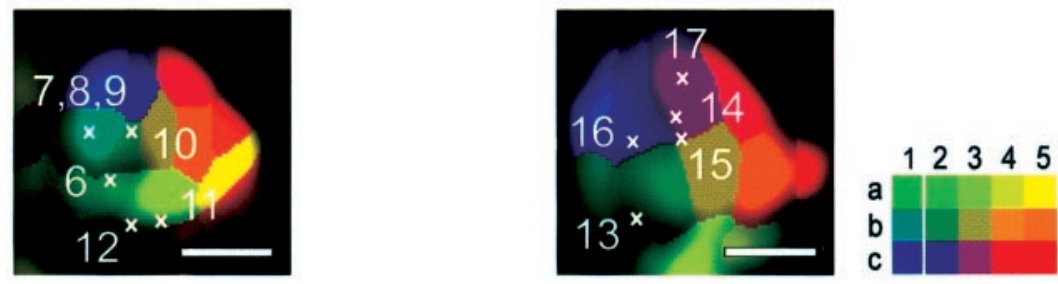

$\mathrm{E}$

6

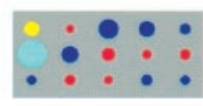

7

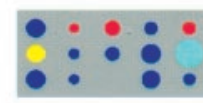

8

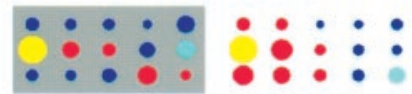

9

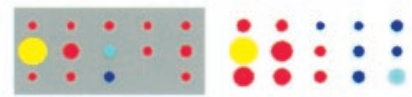

10

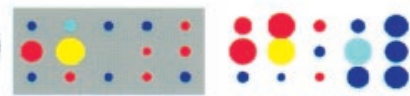

11

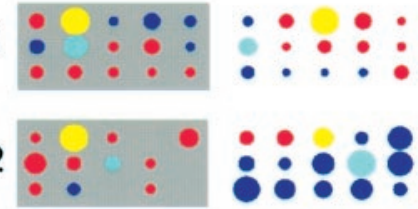

$E$

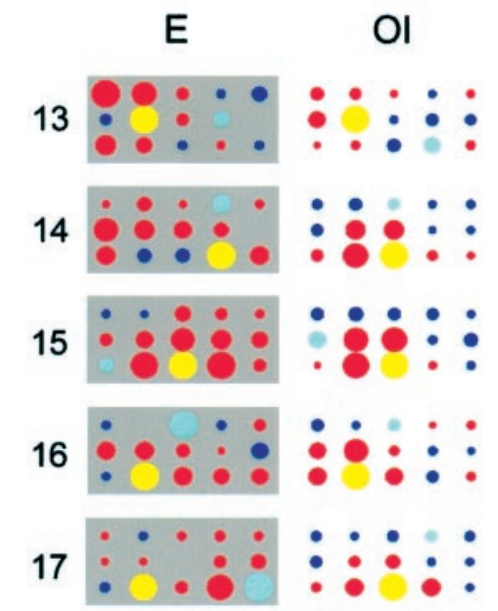

B

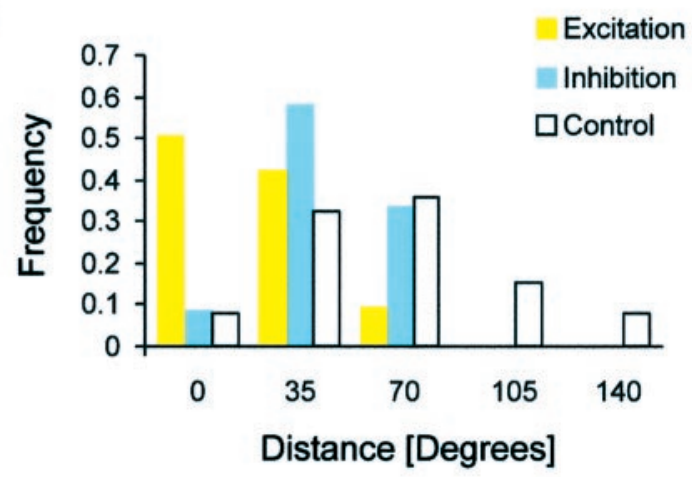

Figure 4. Optical and electrical recording of lateral inhibition. A, In the top row, the track positions are marked in the color-coded retinotopic maps from three animals. The color code for stimulus position used for experiments with combined electrical and optical recordings is shown at the top right. The bottom part of this panel depicts two-dimensional plots of position tuning curves recorded electrically (dark gray background) and optically (light gray background) at the positions of the electrode tracks. Each colored circle displays the response to the corresponding stimulus position. The radius of each circle is proportional to the absolute value of the normalized firing rate or the position-tuning curve reconstructed from the optical signal at the track position. Red (blue) color indicates supra (sub) blank response. The circle corresponding to the stimulus, which elicits maximal (minimal) response, is colored in yellow (turquoise); see also the legend at the bottom left. B. Histogram of the distances between the positions of the peaks of excitation ( yellow bars, $n=22$ ) and inhibition (turquoise bars, $n=17$ ) determined electrically and optically. The control distribution (white bars) was constructed from randomized pairs of positions. Scale bars, $1 \mathrm{~mm}$.

in area 17 , we scaled the maximum intensity projection appropriately. Because, as for inhibition, the intrinsic signal differed markedly between patches evoked by adjacent stimuli, and because of the large overlap of patches in extrastriate areas, we used the average position projection rather than the peak position projection for the color code (see Materials and Methods).
The color-coded retinotopic maps in Figure 5 suggest that a retinotopically organized area is located lateral to area 17 (yellow arrows) in a region probably corresponding to area LM (Olavarria et al., 1982; Olavarria and Montero, 1989). In this area, the lateral part responds to peripheral stimuli, whereas the medial part is activated by central stimuli; i.e., the representation in area 


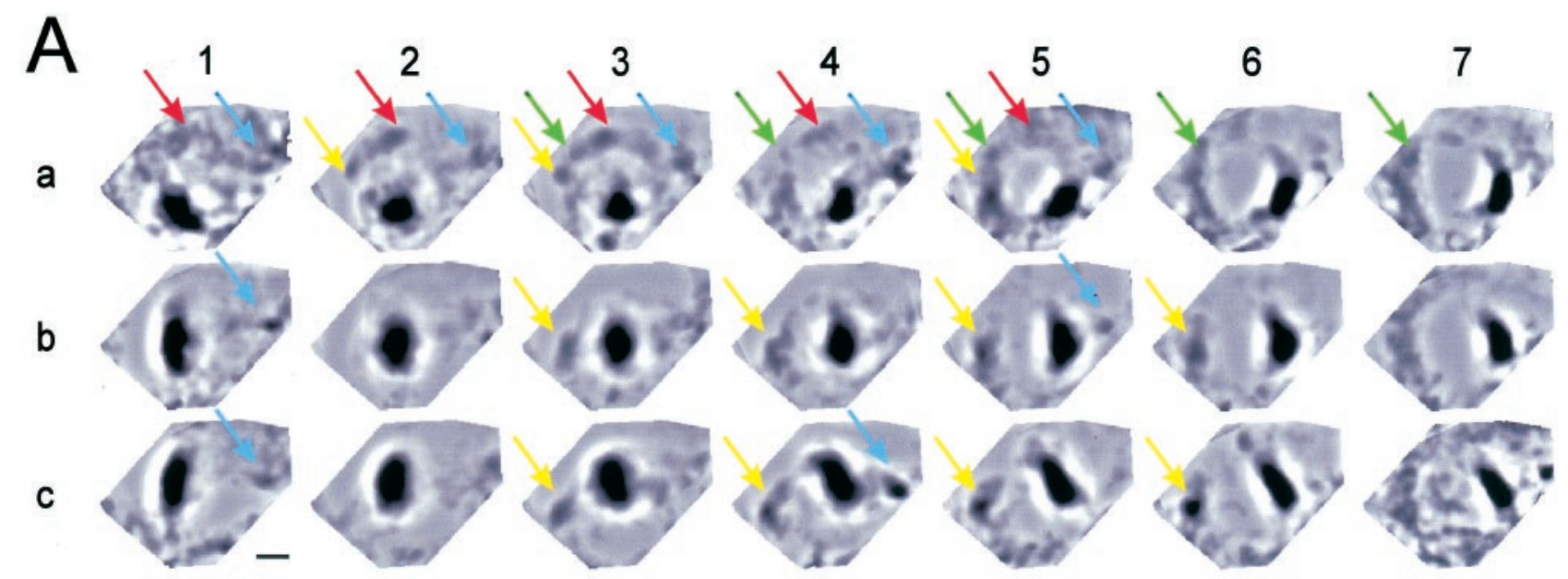

B
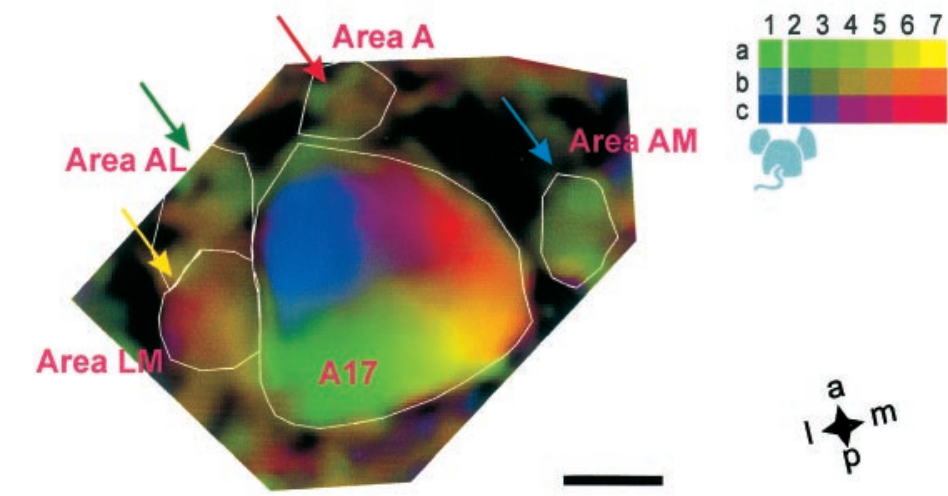

Figure 5. Retinotopic organization of extrastriate visual areas. $A$, High-pass-filtered, averaged single-condition maps $($ boxcar filter $=800 \mu \mathrm{m} ; n=7)$. The colored arrows were placed at the same positions in all maps; they indicate distinct patches in extrastriate areas (yellow: area LM; green: area AL; red: area A; blue: area AM). Note that in many images several extrastriate patches are visible. The pronounced white ring surrounding the dark patch in area 17 was introduced by the strong high-pass filter applied to these maps; it does not indicate the shape of the inhibitory surround. $B$, Color-coded retinotopic map using the high-pass-filtered single-condition maps shown in $A$. The color corresponds to the code for the average position projection Color saturation is proportional to the scaled maximum intensity projection of the high-pass-filtered maps. Candidate extrastriate areas were outlined on the basis of the presence of separate patches in the single-condition maps in $A$. Scale bars, $1 \mathrm{~mm}$.

LM seems to be mirrored at the border to area 17 with respect to the representation in the primary visual cortex, as in other rodents (Montero, 1981, 1993).

From the single-condition maps, we infer the existence of a distinct area anterior-lateral to area 17 (Fig. 5, green arrows, Area $A L$ ) (Olavarria et al., 1982; Olavarria and Montero, 1989) as well as an area anterior to area 17 (red arrows) that may correspond to area A described in rats (Olavarria and Montero, 1984).

We propose that these regions are separate areas, because distinct patches corresponding to each region can be observed in the same single-condition maps. In addition, we observed nonretinotopically organized visual activity (Fig. 5, blue arrows) in a region medial to area 17 , most likely area AM (Olavarria and Montero, 1989).

\section{DISCUSSION}

We have used optical imaging of intrinsic signals to visualize the retinotopic organization of mouse visual cortex. The resulting single-condition maps are highly reproducible and demonstrate that retinotopy is maintained across the entire mouse primary visual cortex. In addition, we observed visually evoked activity in several extrastriate areas. The optically determined maps were confirmed with single-unit recordings, which provided independent evidence for the presence of inhibitory surrounds seen in the imaged maps.

\section{Averaging the intrinsic signal across animals}

Optical imaging of intrinsic signals is based on extensive averaging across multiple trials in individual animals (Grinvald et al., 1986). Because most of the columnar structures, which are routinely imaged in the visual cortex of higher mammals, e.g., orientation or ocular dominance columns, are variable from animal to animal, averaging across animals is impractical for these maps. In contrast, the arrangement and layout of the retinotopic map seem to be rather stable between animals from the same species (Dräger, 1975; Tusa et al., 1978; Wagor et al., 1980; Tootell et al., 1988). In fact, we found a high inter-animal correlation of aligned single-condition maps. Thus, we could reconstruct the retinotopic order directly from maps averaged across animals. We believe that this approach is the most efficient and illustrative way to display data from multiple animals. In particular, one can directly estimate the inter-animal variability of the maps by the corre- 
sponding distribution of the inter-animal SEM per pixel. In addition, averaging maps across animals provides a very intuitive way to illustrate properties, for which automated quantification within the single-condition maps is difficult. In this study, this advantage has proven useful for analyzing the retinotopic structure of extrastriate areas, which would have been very difficult in individual animals. Obviously, low inter-animal variability is a prerequisite for reconstruction of ordered maps using the averaged single-condition maps. Potentially, this approach might also be useful for optical imaging within the barrel cortex.

\section{Interocular alignment of retinotopic maps}

By independently stimulating the ipsilateral and contralateral eyes, we were able to study the organization of ocular preference in mouse visual cortex. We failed to detect any pattern of regions responding preferentially to one eye, implying that unlike in higher mammals, ocular dominance is not mapped in a parcellated manner across mouse visual cortex. Furthermore, we found that the retinotopic maps recorded during stimulation of each eye matched almost completely. This suggests that the retinotopic projections are well aligned between the eyes. Because this alignment could be measured with high precision, optical imaging of mouse visual cortex provides a powerful tool for clarifying the developmental mechanism generating this interocular match.

\section{Cortical magnification factor and ganglion cell density}

It has been hypothesized that the primary central representation of sensory systems scales with the receptor surface (Talbot and Marshal, 1941; Hallett, 1987), i.e., that the distribution of the cell number across the sensory surface matches the distribution of the cell number or area of its neuronal representation. The favorite model to test this hypothesis is the relation between the distribution of the retinal ganglion cell density and the corresponding magnification factor in cortical or subcortical structures. Most of these studies have been performed in higher mammals, which have a fovea or a similar retinal specialization and display a corresponding increase in magnification factor at the foveal representation. Some studies (Schein and de Monasterio, 1987; Wässle et al., 1989) supported the scaling hypothesis, whereas others obtained contradicting results (Azzopardi and Cowey, 1993; Quevedo et al., 1996). Despite the conflicting evidence regarding the match between the distributions of retinal ganglion cell density and area of the neural representation, the peaks of the distributions did coincide in these studies. In mice, ganglion cell density peaks in the central part of the retina, very close to the optic disk (Dräger and Olsen, 1981). However, the CMF peaks in the central region of the visual field, $\sim 55^{\circ}$ away from the optic disk representation, corresponding to a region in the temporal retina $>1 \mathrm{~mm}$ distant from the optic disk, where ganglion cell density has dropped to approximately half of the value in the central retina (Dräger and Olsen, 1981). Thus, in mice the peaks of the vertical and horizontal CMF do not coincide with the peak in retinal ganglion cell density. One possible explanation for this apparent mismatch is that those parts of the cortex representing the central visual field receive inputs from both retinas, thus in effect doubling the number of retinal ganglion cells to be represented in this cortical region. In addition, it is conceivable that in the binocular region of the visual cortex more neuronal circuitry is needed to perform binocular computations.

\section{Optical imaging of inhibition}

The intrinsic signal recorded in optical imaging has been shown to be a metabolic correlate of neural excitatory activity (Grinvald et al., 1986; Frostig et al., 1990). However, it is unclear to what degree this signal also correlates with decreases in neural firing rate below baseline. In studies using related metabolic signals, no conclusive correlation between inhibitory activity and either 2-deoxyglucose mapping (Ackermann et al., 1984; Sharp et al., 1988) or functional magnetic resonance imaging (Waldvogel et al., 2000) could be established.

In mouse visual cortex, extracellular recordings (Mangini and Pearlman, 1980; Simmons and Pearlman, 1983) have shown that visual stimuli elicit lateral inhibition. We were able to image a correlate of this lateral inhibition: in the single-condition maps, a downward deflection of the intrinsic signal surrounds the activated cortical region in a pattern, which is consistent across different animals. Using single-cell recordings, we confirmed that decreases in neuronal firing rate below baseline correlate with decreases of the intrinsic signal. Thus, optical imaging is capable of visualizing the effect of neuronal inhibition. Our results are in agreement with two other studies in which a decrease in the intrinsic signal was shown to correlate with inhibition elicited by epilepsy-inducing agents (Schwartz and Bonhoeffer, 2001) or local stimulation in the visual field (Das and Gilbert, 1995).

Does this correlation hold true for other metabolic brain imaging methods as well? Because we imaged the intrinsic signal at a wavelength of $707 \mathrm{~nm}$, the relative contribution of the lightscattering component is most likely higher than the oxymetry component (Malonek and Grinvald, 1996). This raises the possibility that the decrease in intrinsic signal in this case is not caused by changes in a true metabolic signal but rather in light scattering. Therefore, 2-deoxyglucose mapping as well as functional magnetic resonance imaging based on the oxymetry component might fail to detect this decrease. Lateral inhibition in mouse visual cortex might serve as a model system to further elucidate the differential contributions of the intrinsic signal components associated with inhibition. In addition, optical imaging of inhibition could further our conception of the development and structure of inhibitory circuitry in the cortex.

\section{Extrastriate areas}

Previous studies have been contradictory with respect to the number and structure of extrastriate visual areas in rodents. According to a scheme initially based on recording and tracing experiments performed in the rat by Montero and colleagues (Montero, 1973; Montero et al., 1973a,b), it has been proposed that rodent extrastriate cortex is composed of up to 10 distinct areas, each containing a continuous and mostly complete map of the visual field (Espinoza and Thomas, 1983; Olavarria and Montero, 1984; Thomas and Espinoza, 1987; Coogan and Burkhalter, 1990, 1993; Montero, 1993). On the other extreme, it has been suggested that rodent extrastriate visual cortex consists of just two areas (Kaas et al., 1989; Rumberger et al., 2001) or a single area (Malach, 1989). These authors propose that the patchy connection patterns observed between striate and extrastriate areas reflect a modular composition of the visual cortex, comparable to the situation in primates. Only a few studies have analyzed the organization of extrastriate areas in the mouse. Tracing experiments suggest that mouse extrastriate cortex also contains at least seven distinct areas (Olavarria et al., 1982; Olavarria and Montero, 1989). Using electrophysiological recordings, Wagor et al. (1980) also concluded that mouse extrastriate cortex is composed of multiple areas.

Many of the proposed extrastriate visual areas in the rat are rather small (often $<0.5 \mathrm{~mm}^{2}$ ), and they seem to be even smaller 
in the mouse (Olavarria and Montero, 1989). In addition, neighboring areas often contain mirror symmetric visual field representations, with the same region of the visual field being represented twice in close proximity in the cortex. Thus, given its limited spatial resolution, one cannot expect optical imaging to reveal the retinotopic organization of all of these areas. Despite this, we regularly observed activation in several cortical regions outside of area 17. In the region lateral to area 17, corresponding to the cytoarchitectonic field 18a (Caviness, 1975), we found two visual areas. The posterior one is likely to correspond to area LM (and possibly area LI), whereas the anterior one corresponds to area AL in the scheme of Olavarria and colleagues (Olavarria et al., 1982; Olavarria and Montero, 1989). We found a retinotopic representation only in area LM; area AL does not regularly exhibit any detectable retinotopic order in our maps.

In addition, we found a distinct area anterior to area 17, which has not been described in previous mouse studies (Dräger, 1975; Wagor et al., 1980; Olavarria et al., 1982; Olavarria and Montero, 1989); however, it has been identified in an anatomical study of rat visual cortex (Olavarria and Montero, 1984). We also observed a visually responsive region medial to area 17 , which might correspond to area AM (Olavarria et al., 1982; Olavarria and Montero, 1989). This region also lacked an obvious retinotopic organization.

Taken together, our optical imaging experiments do not support the idea that rodent extrastriate visual cortex consists of just one or two distinct areas, as has been suggested by several authors (Kaas et al., 1989; Malach, 1989; Rumberger et al., 2001). Rather, our results suggest the presence of at least four extrastriate areas in mouse visual cortex, thus giving support to the view that rodent extrastriate cortex consists of multiple distinct areas.

\section{Imaging mouse retinotopy as a tool for the study of transgenic animals}

Genetically altered mice have become a standard tool for assessing the function of different proteins in the mammalian nervous system. By now it is well established that gradients of certain molecules such as ephrins are instrumental in setting up a retinotopic map (Baier and Bonhoeffer, 1992; Cheng et al., 1995; Drescher et al., 1995). Imaging mouse retinotopy now provides a tool for functionally assessing retinotopic maps in such genetically altered mice. Many of the above findings are of great advantage to such investigations. The small inter-animal variance of the intrinsic signal is helpful for this approach because averaging maps across animals provides a tool to detect even subtle differences between experimental groups. In particular, the possibility of statistically testing the optical response per pixel across animals might help to substantiate such differences. Because the position of excitatory patches and the corresponding CMF can be quantified with high precision, these measures are ideally suited for studying the formation of topographic maps in genetically altered mice. Finally, as we have shown, optical imaging provides an efficient tool for mapping extrastriate visual areas in the mouse. Thus, genetic factors influencing areal specification in the cerebral cortex can be easily investigated.

\section{REFERENCES}

Ackermann RF, Finch DM, Babb TL, Engel J (1984) Increased glucose metabolism during long-duration recurrent inhibition of hippocampal pyramidal cells. J Neurosci 4:251-264.

Azzopardi P, Cowey A (1993) Preferential representation of the fovea in the primary visual cortex. Nature 361:719-721.

Baier H, Bonhoeffer F (1992) Axon guidance by gradients of a targetderived component. Science 255:472-475.
Blasdel GG, Salama G (1986) Voltage-sensitive dyes reveal a modular organization in monkey striate cortex. Nature 321:579-585.

Bonhoeffer T, Grinvald A (1996) Optical imaging based on intrinsic signals. The methodology. In: Brain mapping: the methods (Toga AW, Mazziotta JC, eds), pp 55-97. London: Academic.

Bosking WH, Zhang Y, Schofield B, Fitzpatrick D (1997) Orientation selectivity and the arrangement of horizontal connections in tree shrew striate cortex. J Neurosci 17:2112-2127.

Caviness VS (1975) Architectonic map of neocortex of the normal mouse. J Comp Neurol 164:247-263.

Cheng HJ, Nakamoto M, Bergemann AD, Flanagan JG (1995) Complementary gradients in expression and binding of ELF-1 and Mek4 in development of the topographic retinotectal projection map. Cell 82:371-381.

Coogan TA, Burkhalter A (1990) Conserved patterns of cortico-cortical connections define areal hierarchy in rat visual cortex. Exp Brain Res 80:49-53.

Coogan TA, Burkhalter A (1993) Hierarchical organization of areas in rat visual cortex. J Neurosci 13:3749-3772.

Daniel PM, Whitteridge D (1961) The representation of the visual field on the cerebral cortex in monkey. J Physiol (Lond) 159:203-221.

Das A, Gilbert CD (1995) Long-range horizontal connections and their role in cortical reorganization revealed by optical recording of cat primary visual cortex. Nature 375:780-784.

Dräger UC (1975) Receptive fields of single cells and topography in mouse visual cortex. J Comp Neurol 160:269-290.

Dräger UC, Olsen JF (1981) Ganglion cell distribution in the retina of the mouse. Invest Ophthalmol Vis Sci 20:285-293.

Drescher U, Kremoser C, Handwerker C, Loschinger J, Noda M, Bonhoeffer F (1995) In vitro guidance of retinal ganglion cell axons by RAGS, a $25 \mathrm{kDa}$ tectal protein related to ligands for Eph receptor tyrosine kinases. Cell 82:359-370.

Duffy KR, Murphy KM, Jones DG (1998) Analysis of the postnatal growth of visual cortex. Vis Neurosci 831-899.

Espinoza SG, Thomas HC (1983) Retinotopic organization of striate and extrastriate visual cortex in the hooded rat. Brain Res 137-144.

Flanagan JG, Vanderhaeghen P (1998) The ephrins and Eph receptors in neural development. Annu Rev Neurosci 21:309-345.

Frostig RD, Lieke EE, Ts'o DY, Grinvald A (1990) Cortical functional architecture and local coupling between neuronal activity and the microcirculation revealed by in vivo high-resolution optical imaging of intrinsic signals. Proc Natl Acad Sci USA 87:6082-6086.

Girman SV, Sauve Y, Lund RD (1999) Receptive field properties of single neurons in rat primary visual cortex. J Neurophysiol 82:301-311.

Grinvald A, Lieke E, Frostig RD, Gilbert CD, Wiesel TN (1986) Functional architecture of cortex revealed by optical imaging of intrinsic signals. Nature 324:361-364.

Hallett PE (1987) The scale of the visual pathways of mouse and rat. Biol Cybern 57:275-286.

Hubel DH, Wiesel TN (1977) Functional architecture of macaque monkey visual cortex (Ferrier lecture). Proc R Soc Lond B Biol Sci 198:1-59.

Hübener M, Shoham D, Grinvald A, Bonhoeffer T (1997) Spatial relationships among three columnar systems in cat area 17. J Neurosci 17:9270-9284.

Kaas JH, Krubitzer LA, Johanson KL (1989) Cortical connections of areas 17 (V-I) and 18 (V-II) of squirrels. J Comp Neurol 281:426-446.

Malach R (1989) Patterns of connections in rat visual cortex. J Neurosci 9:3741-3752.

Malonek D, Grinvald A (1996) Interactions between electrical activity and cortical microcirculation revealed by imaging spectroscopy: implications for functional brain mapping. Science 272:551-554.

Mangini NJ, Pearlman AL (1980) Laminar distribution of receptive field properties in the primary visual cortex of the mouse. J Comp Neurol 193:203-222.

Metin C, Godement P, Imbert M (1988) The primary visual cortex in the mouse: receptive field properties and functional organization. Exp Brain Res 69:594-612.

Montero VM (1973) Evoked responses in the rat's visual cortex to contralateral, ipsilateral and restricted photic stimulation. Brain Res 53:192-196.

Montero VM (1981) Comparative studies on the visual cortex. In: Cortical sensory organization, Vol 2: multiple visual areas (Woolsey $\mathrm{CN}$, ed), pp 33-81. Clifton, NJ: Humana.

Montero VM (1993) Retinotopy of cortical connections between the striate cortex and extrastriate visual areas in the rat. Exp Brain Res 94:1-15.

Montero VM, Rojas A, Torrealba F (1973a) Retinotopic organization of striate and peristriate visual cortex in the albino rat. Brain Res 53:197-201.

Montero VM, Bravo H, Fernandez V (1973b) Striate-peristriate corticocortical connections in the albino and gray rat. Brain Res 53:202-207.

Olavarria J, Montero VM (1984) Relation of callosal and striateextrastriate cortical connections in the rat: morphological definition of extrastriate visual areas. Exp Brain Res 54:240-252. 
Olavarria J, Montero VM (1989) Organization of visual cortex in the mouse revealed by correlating callosal and striate-extrastriate connections. Vis Neurosci 3:59-69.

Olavarria J, Mignano LR, Van Sluyters RC (1982) Pattern of extrastriate visual areas connecting reciprocally with striate cortex in the mouse. Exp Neurol 78:775-779.

Quevedo C, Hoffmann KP, Husemann R, Distler C (1996) Overrepresentation of the central visual field in the superior colliculus of the pigmented and albino ferret. Vis Neurosci 13:627-638.

Rumberger A, Tyler CJ, Lund JS (2001) Intra- and inter-areal connections between the primary visual cortex V1 and the area immediately surrounding V1 in the rat. Neuroscience 102:35-52.

Schein SJ, de Monasterio FM (1987) Mapping of retinal and geniculate neurons onto striate cortex of macaque. J Neurosci 7:996-1009.

Schuett S, Bonhoeffer T, Hübener M (2000) Mapping of retinotopy in rat visual cortex by combined linear extraction and principal component analysis of optical imaging data. Eur J Neurosci Abstr 12:74.

Schwartz T, Bonhoeffer T (2001) In vivo optical imaging of epileptic foci and surround inhibition in ferret cerebral cortex. Nat Med 7:1063-1067.

Sharp JW, Gonzalez MF, Morton MT, Simon RP, Sharp FR (1988) Decreases of cortical and thalamic glucose metabolism produced by parietal cortex stimulation in the rat. Brain Res 438:357-362.

Simmons PA, Pearlman AL (1983) Receptive-field properties of transcallosal visual cortical neurons in the normal and reeler mouse. J Neurophysiol 50:838-848.

Sperry RW (1963) Chemoaffinity in the orderly growth of nerve fiber patterns and connections. Proc Natl Acad Sci USA 50:703-710.

Talbot SA, Marshal WH (1941) Physiological studies on neural mecha- nisms of visual localization and discrimination. Am J Ophthalmol 24:1255-1264.

Tessier-Lavigne M, Goodman CS (1996) The molecular biology of axon guidance. Science 274:1123-1133.

Thomas HC, Espinoza SG (1987) Relationships between interhemispheric cortical connections and visual areas in hooded rats. Brain Res 417:214-224.

Tootell RB, Switkes E, Silverman MS, Hamilton SL (1988) Functional anatomy of macaque striate cortex. II. Retinotopic organization. J Neurosci 8:1531-1568.

Ts'o DY, Frostig RD, Lieke EE, Grinvald A (1990) Functional organization of primate visual cortex revealed by high resolution optical imaging. Science 249:417-420.

Tusa RJ, Palmer LA, Rosenquist AC (1978) The retinotopic organization of area 17 (striate cortex) in the cat. J Comp Neurol 177:213-235.

Wagor E, Mangini NJ, Pearlman AL (1980) Retinotopic organization of striate and extrastriate visual cortex in the mouse. J Comp Neurol 193:187-202.

Waldvogel D, van Gelderen P, Muellbacher W, Ziemann U, Immisch I, Hallett M (2000) The relative metabolic demand of inhibition and excitation. Nature 406:995-998.

Wässle H, Grunert U, Rohrenbeck J, Boycott BB (1989) Cortical magnification factor and the ganglion cell density of the primate retina. Nature 341:643-646.

Wong-Riley M (1979) Changes in the visual system of monocularly sutured or enucleated cats demonstrable with cytochrome oxidase histochemistry. Brain Res 171:11-28. 